\title{
Eurostudia
}

\section{La laïcité, dispositif d'usage du religieux par les gouvernants? Le cas de la Turquie}

\section{Elise Massicard}

Volume 13, numéro 1-2, 2018-2019

Cultures en contact, entre régulations et représentations

URI : https://id.erudit.org/iderudit/1064488ar

DOI : https://doi.org/10.7202/1064488ar

Aller au sommaire du numéro

\section{Éditeur(s)}

Le Centre canadien d'études allemandes et européennes

ISSN

1718-8946 (numérique)

Découvrir la revue

Citer cet article

Massicard, E. (2018). La laïcité, dispositif d'usage du religieux par les gouvernants? Le cas de la Turquie. Eurostudia, 13(1-2), 49-70. https://doi.org/10.7202/1064488ar

\section{Résumé de l'article}

La République de Turquie présente des modes de gouvernement du religieux spécifique. Le principe constitutionnel de laïcité équivaut à un contrôle par l'État du religieux. Une forme d'islam quasi-officiel est promue par les institutions publiques, prétendant monopoliser le champ religieux, aux détriments d'autres options religieuses. Cependant, ce dispositif n'est jamais parvenu à neutraliser le potentiel politique de la religion. La politisation des questions religieuses, en fait l'un des principaux objets de débat et de lutte politique. L'arrivée au pouvoir de gouvernements dominés par des partis issus de l'islam politique a-t-elle modifié le gouvernement du religieux? Les promesses de libéralisation n'ont pas donné lieu à une plus grande neutralité de l'État par rapport aux interprétations religieuses, malgré les condamnations de la Turquie par la CEDH pour discrimination religieuse. En revanche, les gouvernements du Parti de la Justice et du Développement ont renforcé l'appareil religieux d'État et promu la visibilité de l'islam dans l'espace public. On assiste donc au renforcement des usages de la religion à des fins politiques.
Tous droits réservés $@$ Le Centre canadien d'études allemandes et européennes, 2019
Ce document est protégé par la loi sur le droit d'auteur. L'utilisation des services d’Érudit (y compris la reproduction) est assujettie à sa politique d'utilisation que vous pouvez consulter en ligne.

https://apropos.erudit.org/fr/usagers/politique-dutilisation/ 
La laïcité, dispositif d'usage du religieux par les gouvernants?

Le cas de la Turquie

\section{Elise Massicard}

CNRS / Centre de recherches internationales

\section{Résumé}

La République de Turquie présente des modes de gouvernement du religieux spécifique. Le principe constitutionnel de laïcité équivaut à un contrôle par l'État du religieux. Une forme d'islam quasi-officiel est promue par les institutions publiques, prétendant monopoliser le champ religieux, aux détriments d'autres options religieuses. Cependant, ce dispositif n'est jamais parvenu à neutraliser le potentiel politique de la religion. La politisation des questions religieuses, en fait l'un des principaux objets de débat et de lutte politique. L'arrivée au pouvoir de gouvernements dominés par des partis issus de l'islam politique a-t-elle modifié le gouvernement du religieux? Les promesses de libéralisation n'ont pas donné lieu à une plus grande neutralité de l'État par rapport aux interprétations religieuses, malgré les condamnations de la Turquie par la CEDH pour discrimination religieuse. En revanche, les gouvernements du Parti de la Justice et du Développement ont renforcé l'appareil religieux d'État et promu la visibilité de l'islam dans l'espace public. On assiste donc au renforcement des usages de la religion à des fins politiques. 


\section{Abstract}

The Republic of Turkey presents specific patterns of governing the religious realm. The Constitutional principle of laiklik (secularism) amounts to a control of religion by the state. A form of quasi-official Islam is promoted by public institutions, that have a pretention to monopolize the religious realm to the detriment of alternative religious interpretations. This, however, never managed to neutralize the political potential of religion. On the contrary, the politicization of religious issues leads to religion being one of the most debated and contested issues of political struggles. To what extent has the access to power of parties coming from an Islamist tradition impacted the government of religion? Their promises of liberalizing the religious realm haven't given rise to a more neutral stance of the state toward different religious interpretations, despite the condemnations of Turkey by the European Court of Human Rights on the grounds of religious discrimination. However, the Justice and Development Party in office has fostered the state religious apparatus and promoted the visibility of mainstream Islam in public space. Therefore, one observes growing uses of religion for political purposes.

La République de Turquie proclamée en 1923 constitue la première expérience de régime républicain dans le monde musulman; elle est souvent présentée comme le seul pays «musulman et laïc». Quel destin pour un pays héritier du plus grand Empire musulman au monde, qui a exercé sa domination sur une bonne partie des mondes de l'islam pendant six siècles! L'Empire ottoman n'a pas seulement été le siège plusieurs fois centenaire du califat; l'islam y était religion d'État et le socle de sa légitimation, aussi multiconfessionnel fût-il. C'est dire à quel point cette "laïcité », principe constitutionnel de la République de Turquie depuis 1937, pose question. De fait, elle relève avant tout de modes de gouvernement du religieux spécifiques, qui peuvent se résumer à un contrôle par l'État du religieux. Une forme d'islam quasi-officiel est promue par les institutions publiques, prétendant monopoliser le champ religieux, aux détriments d'autres options religieuses. Cependant, ce dispositif n'est jamais parvenu à neutraliser le 
potentiel politique de la religion; les questions religieuses figurent depuis des décennies au centre des débats et des luttes politiques. Dans quelle mesure l'arrivée au pouvoir de gouvernements dominés par des partis issus de l'islam politique a-t-elle modifié le gouvernement du religieux?

Après avoir dessiné les contours et les tensions de la laïcité en Turquie, nous reviendrons sur deux évolutions récentes du gouvernement du religieux : d'une part, les changements liés au rapprochement avec l'Europe. D'autre part, ceux liés à l'exercice du pouvoir, depuis 2002, d'un parti issu de la mouvance islamiste, l'AKP (Adalet ve Kalkınma Partisi, Parti de la Justice et du Développement). On verra que les promesses de libéralisation qui ont porté ce parti au pouvoir n'ont pas donné lieu à une plus grande neutralité de l'État par rapport aux questions religieuses, malgré les condamnations de la Turquie par la $\mathrm{CEDH}$ pour discrimination. En revanche, les gouvernements de l'AKP ont renforcé l'appareil religieux d'État et promu la visibilité de l'islam dans l'espace public. On assiste donc, paradoxalement, au renforcement des usages de la religion à des fins politiques.

\section{État et religion(s) dans la République de Turquie}

De l'Empire à la République, de la théocratie à la laïcité?

Les souverains ottomans avaient procédé à une institutionnalisation sans précédent de l'islam au sein de l'État. A partir du XVIe siècle, on peut considérer le sunnisme comme religion officielle de l'Empire. Soliman le Magnifique (1520-1566) crée ainsi la fonction de cheikh-ül islam, chef des clercs musulmans (sorte d'équivalent $\mathrm{du}$ patriarche grec orthodoxe). La construction de cette espèce d'Eglise musulmane fortement hiérarchisée, liée à l'État et rétribuée par lui, n'a pas de précédent dans la tradition musulmane sunnite. L'Empire ottoman constituait 
une entité politique de plus en plus sécularisée, l'égalité de tous les sujets étant reconnue depuis le milieu du XIXe siècle.

Mustafa Kemal a voulu instaurer une République en rupture avec cet héritage, reposant sur une tout autre légitimité. Durant les premières années du jeune État, de nombreuses réformes d'ampleur prennent pour cible le religieux. Au niveau institutionnel, le gouvernement républicain abolit le sultanat. Cette mesure équivalait à l'abolition des pouvoirs temporels du chef religieux de l'islam: le calife n'étant plus sultan, il n'avait plus, dès lors, que des attributions spirituelles. Mais en mars 1924, l'Assemblée nationale abolit également le califat, et le dernier calife est expulsé de Turquie. En outre, elle vote une loi qui rattache toutes les institutions d'enseignement au ministère de l'Education nationale, conduisant à la fermeture des écoles coraniques. La position de cheikh-ül islam avait entre-temps été abolie. En 1926, le gouvernement abolit la charia comme source de loi, et les remplace par... une adaptation du code civil suisse. L'article 2 de la première Constitution de la République, celle de 1924, stipule que « la religion de l'État turc est l'islam ». En 1928, cet article est amendé, et la référence à l'islam supprimée; il n'y a plus de religion d'État.

Le terme turc de laïcité est postérieur à ces réformes. Il apparaît à la fin des années 1920 à l'heure des premiers bilans tirés par le régime kémaliste lui-même ${ }^{1}$, pour mettre en cohérence rétrospectivement une série de mesures ayant des champs d'application distincts. Le terme n'apparaît dans le vocabulaire institutionnel qu'en 1937, par son insertion dans la Constitution, à l'occasion de la révision du texte initial, avec les cinq autres grands principes du kémalisme définis en 1931. Cependant, ce terme de laïcité donne souvent lieu à des malentendus. Le parallèle souvent dressé avec la laïcité française est trompeur, même si l'inspiration

\footnotetext{
${ }^{1}$ Lors du discours-fleuve (Nutuk) prononcé par Mustafa Kemal devant l'Assemblée nationale en octobre 1927.
} 
existe bien, puisque le terme « laiklik » est créé en turc moderne à partir du vocable français. En effet, les relations entre État turc et religion sont loin d'être aussi simples qu'il y paraît au premier abord. En réalité, la Turquie ne connaît pas de véritable séparation entre État et religion, ou plutôt il s'agit d'une séparation à sens unique : ces mesures visent à écarter la religion de la vie politique et publique. Si la religion ne doit pas intervenir sur les affaires d'État, en revanche l'État se doit d'intervenir sur les affaires religieuses. Immédiatement après l'abolition du califat, en 1924, est créée une Direction des affaires religieuses, le Diyanet Işleri Başkanlı̆̆ (souvent désigné par DİB ou Diyanet), directement rattachée au cabinet du premier ministre - et depuis 2018 de la Présidence. Cette institution est chargée de réguler et d'administrer toutes les questions de croyance et de rituel musulmans. C'est le Diyanet qui (sauf entre 1931 et 1950) nomme, destitue et procède à la fonctionnarisation des hommes de religion, les imams et muezzins, après avoir surveillé leur formation dans des écoles de prédicateurs. C'est également le Diyanet qui contrôle les mosquées (sauf entre 1931 et 1950), décide des prêches qui y sont lus chaque vendredi, et administre le pèlerinage à La Mecque. Le Diyanet supervise enfin les ouvrages d'enseignement de l'islam. Le Diyanet constitue en somme un imposant appareil religieux destiné à institutionnaliser et domestiquer l'islam. Loin d'avoir coupé les ponts avec la religion, l'État continue donc de l'organiser et de l'enseigner. Producteur de normes religieuses qu'il essaie d'imposer à la société, il se veut le titulaire exclusif des affaires religieuses et l'autorité ultime en la matière. Il ne s'agit donc pas d'un désinvestissement de l'État par rapport au religieux, mais d'un contrôle de la religion par l'État.

\section{Une monopolisation croissante par l'État du religieux}

Cela n'est pas sans rappeler, ironiquement, une tradition ottomane. Sur la longue durée, les réformes kémalistes apparaissent comme une étape dans le processus de domestication de la religion par l'État. On pourrait même affirmer 
que, par rapport à l'Empire ottoman, la prétention de l'État à monopoliser le religieux se renforce sous la République (Bayart 1994 : 381). Cela est lié à deux raisons principales : d'une part, les groupes non-musulmans, si importants sous l'Empire ottoman, ont vu leur importance numérique et sociale décroître drastiquement, ce qui a conduit à une homogénéisation religieuse de la population autour de l'islam. D'autre part, l'État a tenté de restreindre toute expression divergente de l'islam susceptible d'échapper à son contrôle, et de restreindre la diversité religieuse au sein même de l'islam.

Sous l'Empire ottoman, les principaux groupes confessionnels nonmusulmans monothéistes bénéficiaient d'une large autonomie religieuse et légale. Obtinrent le statut de millet les communautés monothéistes reconnues comme «gens du Livre » par l'islam, juifs et chrétiens - avec, pour la chrétienté, ses différentes confessions et Eglises. Ce statut juridique et administratif fut renforcé et institutionnalisé au cours du XIXe siècle. Le traité de Lausanne signé en juillet 1923 a octroyé - sous pression internationale - aux groupes qui avaient auparavant un statut de millet, celui de minorité (azınlık). Ce statut leur garantit les mêmes droits que les citoyens musulmans et leur octroie une série de droits positifs; la liberté d'exercer leur culte et l'accès à toutes les positions dans l'administration. Certains de ces droits seront de fait limités dans la pratique. Ces minorités reconnues ont dès lors le droit de s'organiser en fondations, avoir leurs hôpitaux, leurs écoles où ils peuvent employer leur langue. La rénovation de leurs lieux de culte et les salaires de leurs dignitaires religieux sont à la charge de leur communauté. Ainsi, ces groupes bénéficient d'une reconnaissance et d'un statut sous la République. Mais la jeune République de Turquie ayant repris très largement le code civil suisse en 1926 , les minorités religieuses ont renoncé à une partie des droits spécifiques découlant du Traité de Lausanne (1923), par exemple en matière de juridiction personnelle et familiale (Akgönül 2008 : 181) En outre, les autorités administratives et judiciaires turques ont adopté une interprétation 
restrictive du traité de Lausanne, avec par exemple la privation progressive pour les fondations du droit d'acquérir, d'hériter ou de récupérer des biens immobiliers.

Cependant, les non-musulmans représentent une part décroissante des citoyens : la grande majorité de la population est désormais musulmane, suite au démembrement de l'Empire, aux massacres et aux échanges de population, puis aux vagues d'émigration. Les massacres à la fin du XIXe siècle puis le génocide pendant la Première guerre mondiale ont décimé la population arménienne. Le traité d'échange de population avec la Grèce aboutit à un vaste mouvement de transfert en 1922-1923 des chrétiens orthodoxes de Turquie sauf Istanbul (900 000) vers la Grèce et, inversement, de près de 400000 musulmans de Grèce sauf de Thrace occidentale vers la Turquie. L'impôt sur la fortune dans les années 1940 a, lui, touché les Juifs de plein fouet et beaucoup émigrent en Israël à sa fondation. L'émigration des Juifs se poursuit aujourd'hui, également vers les États-Unis, l'Espagne et le Portugal. Les émeutes de septembre 1955 contre les Grecs à Istanbul qui dégénèrent en massacres et pillages pendant plusieurs jours ont conduit à la fuite de la majorité des Grecs d'Istanbul en Grèce. Le statut de minorité n'a pas suffi à faire taire un sentiment d'insécurité qui refait surface régulièrement. Aucun de ces groupes, concentrés dans les grandes villes et notamment Istanbul, ne représente aujourd'hui plus de quelques dizaines des milliers de personnes². On estime que moins de $1 \%$ de la population est aujourd'hui non-musulmane.

Dans le cadre de la difficile création d'un État-nation avec une population ethniquement et linguistiquement très diverse, mais religieusement de plus en plus homogène, l'islam joue dans les faits le rôle de religion officielle; il occupe une place de première importance dans la définition de l'identité nationale.

\footnotetext{
${ }^{2}$ Quant aux non-musulmans qui n'ont pas de statut reconnu, aucun groupe - orthodoxes syriaques ou arabophones, assyro-chaldéens, nestoriens - n'atteint la dizaine de milliers de personnes.
} 
C'est également ce contexte historique de création d'un État-nation qui donne sens à la seconde dimension de la monopolisation du religieux par l'État. Si l'islam acquiert un rôle de ciment de la nation, c'est dans le cadre d'un projet de construction d'une communauté nationale qui a pour particularité son caractère unitaire. Par conséquent, l'islam officiel ignore la diversité confessionnelle, pourtant bien réelle, de la Turquie - en n'accordant aucune place aux alévis ou aux yézidis. En pratique, si le kémalisme a souvent eu recours aux références islamiques comme source de légitimation, c'est toujours au sunnisme hanéfite qu'il a fait appel, l'érigeant ainsi en « confession officielle par défaut » (Bozarslan 1994).

Les alévis sont un groupe hétérodoxe et syncrétique qui constitue une proportion non négligeable de la population du pays - entre 10 et $25 \%$ selon les estimations. En raison des éléments d'origine chiite de leur culte (adoration d'Ali, jeûne du mois de muharrem, etc.), les alévis sont parfois assimilés à des adeptes du chiisme. Mais ils suivent une interprétation ésotérique de l'islam et se distinguent par des pratiques spécifiques (cérémonies, dignitaires religieux, culte des pierres, etc.) auxquelles il est difficile d'attribuer une origine précise, et dont certaines sont probablement antérieures à l'islam. De ce fait, l'attitude des musulmans orthodoxes oscille entre leur dénier la qualité même de musulmans et les assimiler à l'islam orthodoxe en niant leur spécificité ou, au contraire, en rapportant cette dernière à des « déviances ». Les autorités - pour qui l'alévité représente tout au plus un phénomène culturel - nient sa spécificité ou la traitent sur un mode folkloriste.

La «laïcité » ne signifie donc ni l'égalité, ni même la reconnaissance de la pluralité confessionnelle. Elle n'implique pas non plus une neutralité de l'État par rapport à la religion mais, au contraire, l'officialisation, en pratique, d'une version précise de l'islam. Là aussi, on constate une continuité avec l'Empire ottoman, qui reconnaissait certes la diversité religieuse, mais uniquement pour les non- 
musulmans, en excluant les musulmans hétérodoxes, dont la différence n'était ni reconnue ni institutionnalisée.

En outre, l'État républicain a tenté de restreindre toute expression divergente de l'islam susceptible d'échapper à son contrôle. Ainsi, en 1925, suite à une révolte dirigée par Sheikh Saïd, de la puissante confrérie nakshibendie dans le sud-est kurdophone du pays, toutes les confréries musulmanes sont interdites et leurs couvents fermés. Or, ceux-ci constituaient d'importants réseaux de transmission et de socialisation religieuse. Dans le même mouvement, le gouvernement interdit le culte des saints et ferme plus de 900 mausolées de saints, stigmatisés comme des lieux de pratiques magiques et superstitieuses, donc opposés au « progrès » et à la «civilisation». Ainsi, l'islam devait être celui professé par l'État - national, modernisé, débarrassé de ses éléments « rétrogrades » ou « obscurantistes »- ou ne pas être. L'islam ne devait pas être éradiqué, mais vidé de tout potentiel d'opposition et même de mobilisation. Dans une large mesure, cette domestication de la religion impliquait son instrumentalisation par le régime kémaliste pour affermir la cohésion nationale.

\section{La politisation de l'islam}

\section{Une monopolisation étatique en déclin}

En revendiquant ce monopole sur l'expression publique de l'islam, l'État turc voulait neutraliser la face publique de la religion, la rendre consensuelle et confiner la croyance à une question privée. Or, en officialisant une version précise de l'islam et réprimant les autres, l'État a abouti à tout, sauf à une neutralisation de l'islam dans l'espace public ou une dépolitisation de la question religieuse. Au contraire, les relations entre l'État et l'islam sont devenues un enjeu très politisé.

De fait, le Diyanet et l'islam officiel ne sont jamais parvenus à exercer le monopole qu'ils revendiquaient sur l'interprétation de l'islam. Violemment 
combattus aux débuts de la République, les courants musulmans alternatifs ont été poussés vers la clandestinité. Les confréries se sont camouflées pour éviter la répression, mais n'ont pas disparu. Certaines ont survécu au prix de l'adaptation de quelques-unes de leurs pratiques à la clandestinité (Zarcone 2004). De nouvelles communautés religieuses issues de milieux confrériques, parfois qualifiées de néoconfréries, se sont formées et implantées avec succès au sein des populations principalement urbaines. Si elles refusaient tout affrontement direct avec le pouvoir, elles répondaient à un régime qui monopolisait l'ensemble de l'espace de visibilité par la formation de tissus de relations sociales et de réseaux de solidarité. Ainsi, le courant nurcu, fondé dans les années 1930 par Said Nursi, un religieux kurde, qui regroupait notamment les classes moyennes de sensibilité religieuse, s'imposa comme une véritable société parallèle que le pouvoir ne parvint jamais à démanteler. A partir des années 1960, la mouvance Fethullahcl, du nom de l'imam Fethullah Gülen qui l'a créée, lui-même issu du courant nurcu, se développe de manière massive. Tentant de concilier croyance et science, éducation et religion, elle s'implante de manière massive dans de nombreux secteurs de la société, rassemblant plusieurs millions d'adeptes et de sympathisants.

L'islam n'a donc jamais vraiment été monopolisé par l'État en Turquie. On peut même affirmer que ce dernier a été de moins en moins régulé par l'État. De nombreux facteurs ont relativisé ce monopole étatique. Mentionnons deux périodes importantes de ce point de vue : la libéralisation des années 1950-1960, puis celle des années 1980-1990.

Le passage au multipartisme en 1950 entraîne une première libéralisation : le parti kémaliste, jusqu'alors parti unique, voire parti-État, se retrouve confronté à la concurrence d'autres orientations politiques. Dans ce contexte, la religion devient un argument électoral de poids, une forme de concession aux demandes sociales utilisée par de nombreux partis, notamment la droite conservatrice. 
Depuis, les relations entre État et religion n'ont pas cessé d'être âprement discutées dans le débat public : la place de l'islam dans l'État et la société, l'existence et le financement de l'enseignement religieux, la place de la religion dans le système scolaire public, le port du voile ont été objets de débats virulents entre courants politiques, mais aussi de luttes entre institutions : armée et justice se faisant les principaux défenseurs d'une conception restrictive, peu tolérante de la laïcité.

Un second tournant a lieu après le coup d'État militaire de 1980. L'armée turque, pour contrer l'extrême politisation de la société et rétablir la stabilité, a de nouveau favorisé l'islam comme ciment national. La Constitution de 1982 introduit ainsi l'enseignement religieux obligatoire dans les établissements scolaires du primaire et du secondaire. Après la remise du pouvoir aux civils, sous l'impulsion d'un parti conservateur et libéral dans les années 1980, une tolérance accrue se manifeste à l'égard des associations religieuses, des confréries et néo-confréries; le port du foulard se fait de plus en plus visible. Cet essor de l'islam dans l'espace public nourrit une polarisation entre « laïcs » et « musulmans », avec des moments de « crise » autour de questions comme le port du voile à l'université, la présence de femmes voilées lors des réceptions officielles, etc.

L'essor, puis l'arrivée au pouvoir de partis islamistes, à partir des années 1990, constitue un tournant. Dans quelle mesure cet accès au pouvoir a-t-il modifié la gestion du religieux par les pouvoirs publics? Cet accès au pouvoir s'est fait de manière progressive, et ce n'est qu'avec la longue expérience de l'AKP au pouvoir, sans coalition, depuis 2002, que l'on peut évaluer ses effets sur la gestion du religieux.

Pour comprendre les circonstances de cet exercice du pouvoir, il convient cependant de revenir sur la trajectoire d'ascension en politique des partis islamistes en Turquie. Des partis islamistes légaux ont été créés dès la fin des années 1960. Malgré la répression institutionnelle, ils ont accepté le jeu parlementaire dans 
lequel ils se sont par la suite imposés. Ils ont gagné un poids électoral de première importance depuis les années 1990. C'est en Turquie que, pour la première fois dans la région, une formation islamiste est arrivée au pouvoir par la voie parlementaire, sans avoir recours à la violence. En 1996, un parti islamiste, le Parti de la Prospérité (RP ou Refah Partisi), sorti premier des législatives de 1995, prenait la tête d'un gouvernement de coalition. Mais cette expérience fut de courte durée : en février 1997, par un mémorandum resté célèbre et qualifié par la presse de « coup d'État post-moderne », les militaires obligeaient le gouvernement à prendre des mesures drastiques envers toutes les manifestations de la « réaction religieuse » (enseignement coranique, associations pieuses, écoles privées d'orientation religieuse, capital islamique, mosquées non contrôlées par le Diyanet, etc.). Quelques mois plus tard, le gouvernement était poussé à la démission et le RP fut fermé par la justice. Courte, contrainte et avortée, cette première expérience au pouvoir ne permet pas au RP de modifier la gouvernance du religieux. Ce sera très différent pour l'expérience suivante du pouvoir, nettement plus longue. En 2002, l'AKP, issu de cette même tradition islamiste arrive aux commandes, cette fois avec une large majorité parlementaire; il se maintient au pouvoir depuis, soit pour plus de quinze ans.

\section{Une promesse déçue de libéralisation}

Cependant, l'AKP n'est pas un parti islamiste comme ses prédécesseurs. La douloureuse expérience du RP au pouvoir a en effet provoqué une transformation profonde de l'islam politique en Turquie. Fondé en 2001, l'AKP procède à un aggiornamento par rapport à cette tradition islamiste dont il est issu. Il adopte une ligne différente, qui se caractérise par l'acceptation des règles du jeu politique et institutionnel. Il ne se présente pas comme parti islamiste ni même musulman, mais comme démocrate-conservateur et libéral : c'est-à-dire comme partisan du «moins d'État », d'une force publique moins présente et directive, et défenseur de 
la liberté sous toutes ses formes. Le port du voile dans les services publics et à l'université a longtemps été proscrit en Turquie, même si aucun texte ne l'interdisait spécifiquement. Pendant des années, les juges - très kémalistes estimaient que cette interdiction résultait du principe constitutionnel de laïcité. $\mathrm{Au}$ contraire, l'AKP considère ce type d'interdictions religieuses en termes de questions de liberté de croyance et de culte. Dans cette perspective, il adopte un positionnement très pro-européen, alors que ses prédécesseurs étaient opposés à l'intégration de la Turquie à l'Union européenne, que le leader du RP considérait comme un « club chrétien ». Pour l'AKP, l'ancrage à l'UE doit permettre de pousser cet agenda de libéralisation - sur les questions religieuses comme sur d'autres -, de surmonter certains blocages institutionnels, et de faire baisser la pression des militaires. D'ailleurs, le rapprochement avec l'Union européenne constituait la priorité du gouvernement de l'AKP dans la première moitié des années 2000. Pourtant, l'agenda de libéralisation avec lequel l'AKP est arrivé au pouvoir, ne le conduit pas à mettre en place une libéralisation du gouvernement du religieux en Turquie, ou seulement de manière très partielle.

L'AKP est arrivé au pouvoir porté par un électorat large et diversifié : conservateurs et religieux, mais aussi libéraux. Lors de ses premières années au pouvoir, il renonce à remettre les questions religieuses, très clivantes, et dont il sait attirer l'ire des militaires, à l'ordre du jour. Le gouvernement évite la censure du juge mais aussi l'affrontement direct par la loi; dans le même temps, l'opposition laïque, consciente de l'attachement de la majorité de la population aux traditions religieuses, devient plus prudente. Le gouvernement défend alors une régulation du religieux moins stricte que la conception restrictive défendue par les militaires,qui perdent une grande partie de leur pouvoir au cours de cette période, sous l'impulsion conjointe de l'AKP et des institutions européennes. Le gouvernement promeut sa position en matière religieuse en termes de libéralisation. Ainsi, il tente de revenir sur l'interdiction du port du voile à l'université au nom de la liberté 
religieuse. Fort de ce cadrage, il espère obtenir l'aide de l'Union européenne, mais aussi de la Cour européenne des Droits de l’Homme

De fait, la CEDH intervient de plus en plus dans les contentieux ayant pour enjeu les relations entre État et religion depuis les années 1990, en défendant les principes de liberté de culte et de croyance 3 . Le processus d'adhésion de la Turquie à l'UE, entamé en 2005 a donné à la jurisprudence de la CEDH un poids politique accru. Mais l'AKP, qui espérait de la Cour un soutien contre l'exclusion de la religion de l'espace public - notamment l'interdiction du port du voile à l'université -, a été déçu. Dans de nombreuses affaires, la Cour s'est rangée du côté de la défense de la laïcité officielle. Concernant le port du foulard à l'université, déjà en 1993 dans l'arrêt Karaduman c. Turquie ${ }^{4}$, après avoir relevé l'existence d'un enseignement privé parallèle à l'enseignement public, la $\mathrm{CEDH}$ avait admis l'interdiction $\mathrm{du}$ port de signes religieux dans les établissements publics d'enseignement supérieur turcs si celui-ci nuit à l'objectif de protection des droits et des libertés d'autrui, de l'ordre et de la sécurité publique. Mais la décision marquante en la matière - car elle intervient en 2005, c'est-à-dire au début de la législature $\mathrm{AKP}$, et au moment où le principe d'ouverture des négociations d'adhésions de la Turquie à l'UE est admis - est l'arrêt Şahin ${ }^{5}$. Il déboute une étudiante qui avait saisi la CEDH en 1998 au motif que l'interdiction du port du foulard islamique à l'université constituerait une violation des droits et libertés. La Cour juge conforme à la Convention européenne des droits de l'homme la conception de la laïcité telle qu'exprimée par l'arrêt de la Cour constitutionnelle

\footnotetext{
${ }^{3}$ En tant que membre du Conseil de l'Europe, la Turquie ratifie la Convention européenne des droits de l'homme en 1954, mais ne ratifie cependant pas tous les protocoles suivants. Elle n'accepte le droit de recours individuel qu'en 1987 et ne reconnaît la juridiction obligatoire de la CEDH qu'en 1990. En partie en raison de ces évolutions, les requêtes déposées contre l'État turc, inférieures à la centaine annuelle jusqu'en 1991, augmentent en flèche dans les années 1990.

${ }^{4}$ CEDH, Commission (Plénière) du 3 mai 1993, Karaduman c. Turquie, n¹6278/90.

${ }^{5} \mathrm{CEDH}$, Arrêt (Grande chambre) du 10 novembre 2005, Şahin c. Turquie, n44774/98.
} 
turque du 7 mars 1989, selon laquelle elle constitue le garant des valeurs démocratiques et des principes d'inviolabilité de la liberté de religion pour autant qu'elle relève du for intérieur et de l'égalité des citoyens devant la loi. La CEDH estime également que la sauvegarde du principe de laïcité peut être considérée comme nécessaire à la protection du système démocratique de la Turquie. Elle estime en outre que l'on ne saurait faire abstraction de l'impact que peut avoir le port du foulard (perçu comme une obligation religieuse contraignante) sur ceux et celles qui ne le portent pas. Une limitation en la matière pourrait donc répondre à un «besoin social impérieux » pour atteindre les deux buts légitimes que sont la laïcité et les droits des femmes, d'autant plus que ce symbole religieux a acquis, au cours de ces dernières années en Turquie, une portée politique. La Cour conclut que la restriction du port du foulard islamique à l'université est justifiée dans ses principes et proportionnée aux buts poursuivis et qu'elle ne viole pas l'article 9 de la Convention. L'approche de la Cour européenne repose sur une reconnaissance des traditions de chaque pays, sans chercher à imposer un modèle uniforme de relations entre État et religion à travers l'Europe. De ce point de vue, la CEDH n'a pas œuvré à un affaiblissement des restrictions des expressions religieuses dans la sphère publique.

Pour modifier la gestion publique du religieux, et ce qu'il considère comme des limitations à l'expression publique de l'islam, l'AKP se trouve donc sans appui de la CEDH et confronté aux résistances de certaines institutions (judiciaires et militaires notamment) encore dominées par des kémalistes jusqu'à la fin des années 2000. Ainsi, la Cour constitutionnelle a-t-elle retoqué, en 2008, un amendement constitutionnel soutenu par le gouvernement qui reconnaissait l'égal accès de tous aux services publics, au motif qu'il violait le principe de laïcité, car il ouvrait la voie à l'autorisation du port du voile. Pour modifier la situation, l'AKP, au fur et à mesure que son contrôle sur la justice s'étendait est donc passé par d'autres moyens : non des lois, susceptibles de provoquer des montées de bouclier, 
mais des décrets ou des circulaires. En 2008, le gouvernement ainsi ordonnait par décret aux recteurs de tolérer les étudiantes voilées dans leurs établissements. En 2013, le gouvernement a levé l'interdiction du voile sur les lieux de travail pour les employées de la fonction publique. Depuis 2013, des députées voilées siègent au Parlement et le voile n'est plus interdit dans les collèges et lycées depuis 2014. Toutes ces mesures ont été justifiées en termes de liberté religieuse, alors qu'on estime qu'environ deux tiers des femmes portent le voile en Turquie.

Au-delà de la visibilité de la religion dominante dans l'espace public, un autre enjeu de la gestion publique du religieux est la diversité et l'égalité en matière religieuse - domaine dans lequel la CEDH s'est avérée très active, mais qui était moins prioritaire dans l'agenda de libéralisation religieuse porté par l'AKP. Dans un premier temps, le gouvernement de l'AKP a donné des signes forts en direction des non-musulmans, dossier sur lequel l'Union européenne a été très proactive. Ainsi, l'AKP a permis la rétrocession de certaines propriétés à des communautés religieuses non-musulmanes. En 2008, l'orphelinat grec sur l'île de Büyükada est redevenu la propriété du Patriarcat œcuménique après une décision de la Cour européenne des droits de l'homme ${ }^{6}$. Avec l'assouplissement de la loi sur les fondations en 2008, les fondations religieuses non-musulmanes peuvent désormais vendre et acheter des biens fonciers ou immobiliers, réparer leurs propriétés, ouvrir des commerces, recevoir des financements et des dons de l'étranger. Malgré la rétrocession de 500 biens immobiliers aux minorités, la réforme ne règle pas totalement la question des propriétés saisies par l'État après 1974 (crise de Chypre). En outre, certains dossiers restent litigieux, notamment la réouverture $\mathrm{du}$ séminaire orthodoxe de Halki.

Ainsi, ces inflexions dans le sens d'un meilleur traitement des autres groupes religieux ne s'accompagnent pas de reconnaissance de la diversité dans l'islam, ce

${ }^{6}$ CEDH, Arrêt du 8 juillet 2008, Patriarcat CEcuménique c. Turquie, n¹4340/05. 
qu'illustre le cas des alévis. Le gouvernement de l'AKP a lancé une initiative appelée « ouverture alévie », destinée à créer, pour la première fois, un dialogue institutionnalisé entre officiels et alévis. Cette série de rencontres, largement critiquée notamment au sujet du choix de ses participants, n'a donné lieu qu'à des mesures symboliques. Pour le reste, la reconnaissance d'un statut religieux pour les alévis a été abandonnée, ce que montre, par exemple, le refus répété et constant d'octroyer aux lieux de réunions religieuses des alévis, les cemevi, le statut de lieux de culte. Leur conférer ce statut impliquerait la reconnaissance d'une pluralité dans l'islam, voire l'acceptation de l'alévité comme culture spécifique. De même, les demandes (non consensuelles parmi les alévis eux-mêmes) que les dignitaires religieux alévis soient rémunérés sur le budget du Diyanet sont restées lettre morte.

À plusieurs reprises, la CEDH a condamné la Turquie pour discrimination religieuse. En 2007, la CEDH a condamné la Turquie au motif que l'enseignement obligatoire de la religion à l'école porte essentiellement sur le sunnisme et que le requérant alévi, à la différence des non-musulmans, ne pouvait pas dispenser ses enfants de ces cours obligatoires ${ }^{7}$. Concernant l'enseignement religieux à l'école, la Turquie n'a pas réellement tiré les conclusions des arrêts de la CEDH. Loin de faire de l'enseignement existant un cours d'éducation religieuse généraliste, ce dernier conserve sa dimension confessionnelle; quelques modules concernant l'alévité ont été ajoutés, et certains enfants exemptés (Massicard 2014). Ainsi, bien que les recours individuels auprès de la $\mathrm{CEDH}$ en termes de discrimination religieuse se soldent presque systématiquement par des condamnations de la Turquie, la promesse de libéralisation de l'AKP n'a pas entraîné une plus grande égalité de traitement entre les différentes croyances religieuses, notamment au sein de l'islam. L'AKP au pouvoir a donc modifié la gestion publique du religieux, mais pas strictement selon l'agenda de libéralisation religieuse qu'il avait annoncé. Il

${ }^{7}$ CEDH, Arrêt du 9 janvier 2008, Hasan et Eylem Zengin c. Turquie, n¹448/04. 
s'agit plutôt d'une libéralisation des expressions publiques de la religion dominante.

La religion comme outil de politiques publiques

Parallèlement, l'AKP n'a pas limité la mainmise de l'État sur la religion; au contraire, il a renforcé les outils de promotion et de diffusion de l'islam officiel, notamment le Diyanet, mais aussi les écoles d'imams-prédicateurs. Depuis son accès au pouvoir, le Diyanet a connu une extension sans précédent. Néanmoins, son essor avait déjà commencé, selon une chronologie qui se rapproche de celle des périodes de libéralisation religieuse évoquées plus haut et qui correspond en grande partie à des périodes dominées par des gouvernements conservateurs. Ses fonctions sont élargies par la loi n633 adoptée en 1965 qui lui confie celle d'éclairer la société à propos de la religion musulmane et ses principes moraux. Le DIB commence dès lors à publier des ouvrages et une revue, et à diffuser toute une littérature de vulgarisation et de sensibilisation sur des thèmes religieux. Il donne son avis pour l'importation d'ouvrages religieux et développe des centres d'apprentissage de la lecture coranique.

Parallèlement à l'élargissement de ses fonctions, cette direction - simple bureau de quelques milliers de personnes à sa création - n'a cessé de connaître une expansion en termes d'effectifs mais aussi de budget. Cette évolution est particulièrement nette durant la décennie 1980, durant laquelle ses effectifs doublent pratiquement, passant de 43197 en 1981 à 74789 en 1991 (Çakır et Bozan 2005 : 74). Mais cette évolution est également très nette après l'arrivée au pouvoir de l'AKP. De 2003 à 2013, le nombre de ses salariés double de nouveau. De 2006 à 2017, le nombre de prédicateurs a doublé, passant de 1210 à 2 487. De même, entre 2003 et 2017, plus de 11000 nouvelles mosquées ont été ouvertes, soit une 
augmentation de plus de $15 \%{ }^{8}$. La part du Diyanet dans le budget public a également doublé, passant de $0,61 \%$ en 2003 à 1,27\% à 2014. Le Diyanet figure ainsi parmi les institutions qui ont été le plus privilégiées par le gouvernement de l'AKP.

Avec le développement d'une migration de Turquie dans les pays européens amenée à perdurer, des représentants du Diyanet ont été envoyés dans les grandes villes de migration turque en Europe. Depuis le milieu des années 1980, le Diyanet dispose de représentants dans les ambassades et les consulats. En 2003, il y avait 14 conseillers, 20 attachés et 1153 fonctionnaires religieux dans 20 pays (Çakır et Bozan 2005 : 92). Cette implantation internationale connaît un essor important sous les gouvernements de l'AKP. Fin 2010, il y avait plus de 40 conseillers et attachés, et 1500 imams envoyés par le Diyanet dans 80 pays (DIB 2011: 77-78) Fin 2017, le Diyanet était actif dans plus de 100 pays (DIB 2018: 34).

En outre, une loi de 2010 a modifié la structure institutionnelle et organisationnelle du Diyanet, et augmenté ses moyens et l'échelle de ses activités au-delà des mosquées et des cérémonies religieuses. Cette loi octroie au Diyanet de nombreuses prérogatives supplémentaires, telles que l'ouverture d'une chaîne radiophonique ou télévisée, et la mission d'éclairer le peuple sur la religion endehors des mosquées. Notamment après l'entrée en vigueur de la nouvelle loi, le nombre des cours coraniques et de leurs élèves a augmenté de manière très sensible. Le nombre de cours de Coran proposés a quadruplé entre 2006 et 2017. A en outre été supprimée en 2011 la limite d'âge pour les cours coraniques - qui s'adressaient jusque-là uniquement aux élèves à partir du collège. Ont ainsi été ouverts des cours coraniques pour les enfants entre quatre et six ans. En 2013, le nombre de ces élèves s'élevait à plus d'un million, soit dix fois plus que dix ans auparavant. On estime qu'aujourd'hui les inscrits aux cours coraniques sous leurs

\footnotetext{
${ }^{8}$ Voir les rapports d'activité annuels du Diyanet, ainsi que les statistiques publiées par l'institution; http://www.diyanet.gov.tr/tr-TR/Kurumsal/Detay//6/diyanet-isleri-baskanligi-istatistikleri.
} 
différentes formes atteignent les cinq millions (DIB 2018 : 55-56). Ainsi, loin d'avoir simplement levé les obstacles à la présence de l'islam dans l'espace public, les gouvernements de l'AKP ont considérablement renforcé le Diyanet comme outil de diffusion d'un islam d'État dans la société, ce qui s'éloigne de l'agenda de libéralisation qu'ils annonçaient à leur arrivée au pouvoir.

\section{Conclusion}

La volonté de neutralisation de la dimension publique et du potentiel politique de la religion portée par les kémalistes s'est avérée lettre morte. Le gouvernement du religieux reste un objet de débats et de luttes en Turquie. Alors que les partisans d'une stricte laïcité insistent sur la neutralité d'une sphère publique non confessionnelle, les islamistes s'attachent à la liberté de conscience et de religion pour lever les limites à l'expression de la foi dans l'espace publique. Mais l'égalité religieuse des citoyens, elle, n'est prioritaire ni pour les premiers, ni pour les seconds. De ce point de vue, la jurisprudence de la CEDH, très active en termes de discrimination religieuse, fournit une autre voie, mais qui a peu de conséquences en interne.

Le gouvernement du religieux en Turquie est marqué par de nombreux paradoxes, incarnés par le Diyanet: censée veiller à l'apolitisme de l'islam, cette institution est aussi critiquée comme vecteur de pénétration des islamistes dans l'appareil d'État et d'islamisation de la société. Certains militants de l'islam politique voient en lui une structure disponible qui attend d'être conquise, et certains défenseurs de la laïcité y voient un garde-fou salutaire contre un islam moins contrôlé. De l'autre côté, certains intellectuels laïcs et certains militants islamistes se rejoignent pour réclamer sa suppression, pour séparer État et religion de manière plus stricte ou, au contraire, pour limiter la tutelle que les institutions exercent sur l'islam et agrandir la marge d'autonomie de celui-ci. 
Sans remettre en cause le principe constitutionnel de laïcité, le gouvernement de l'AKP a considérablement élargi les missions et les moyens du Diyanet. Il a supprimé l'interdiction du port du voile à l'université et la limitation d'âge pour les cours coraniques, qui étaient largement considérés comme les plus importantes manifestations de l'exclusion de l'islam de la sphère publique. Il a ainsi non pas modifié l'inégalité de traitement des différentes religions, mais permis, voire encouragé, l'expression de l'islam sunnite dans la sphère publique, tout en investissant l'appareil religieux d'État d'un rôle accru, conformément au projet de former une « jeunesse pieuse ». L'usage par les gouvernants de l'islam à des fins politiques, remontant aux débuts de la République, reste donc d'actualité. Cette laïcité pourrait donc paradoxalement constituer un dispositif facilitant l'usage du religieux par les gouvernants.

\section{Bibliographie}

Akgönül, S. (2008). « Minorités non-sunnites en Turquie et laïcité », Dans Laïcité en débat: Principes et représentations en France et en Turquie. Strasbourg, Presses universitaires de Strasbourg, p. 171-186.

Akgönül, S. et A. E. Öztürk (dir.). (2018). «Religion as a foreign policy tool». European Journal of Turkish Studies, vol. 27.

Bayart, J.-F. (1994). « Les trajectoires de la République en Iran et en Turquie : un essai de lecture tocquevillienne». Dans G. Salamé (dir.), Démocraties sans démocrates. Paris, Fayard, p. 373-394.

Bozarslan, H. (1994). «Au-delà de l'abolition du Khalifat. Laïcité, État-Nation et contestation kurde ». Les annales de l'autre islam, n`2, p. 225-235. 
Çakır, R. et I. Bozan. (2005). Sivil, Şeffaf ve Demokratik Bir Diyanet İşleri Başkanlığ Mümkün Müdür?. İstanbul, Tesev Yayınları.

DİB. (2011). Diyanet Hizmetler ve Projeler 2003-2010. Ankara, Diyanet İşleri Başkanlığı Yayınları.

DİB. (2018). Faaliyet Raporu 2017. Ankara, Strateji Geliştirme Başkanlığ1.

Massicard, E. (2014). «La judiciarisation contrastée de la question alévie, de la Turquie à l'Europe ». Revue française de science politique, vol. 64, n²4, p. 711733.

Öztürk, A. E. (2016). «Turkey's Diyanet under AKP Rule: from Protector to Imposer of State Ideology? ». Southeast European and Black Sea Studies, vol. 16, $\mathrm{n}^{\circ} 4$, p. 619-635.

Zarcone, T. (2004). La Turquie moderne et l'islam. Paris, Flammarion. 